\title{
The Formula for Health and Well-Being in Individuals With Cerebral Palsy: Cross-Sectional Data on Physical Activity, Sleep, and Nutrition
}

\author{
Patrick G. McPhee, $\mathrm{PhD}^{1,2,3}$, Olaf Verschuren, $\mathrm{PhD}^{4}$, Mark D. Peterson, PhD, $\mathrm{MS}^{5}$, \\ Ada Tang, $\mathrm{PT}, \mathrm{PhD}^{2}$, Jan Willem Gorter, $\mathrm{MD}, \mathrm{PhD}^{1,2,3}$ \\ ${ }^{1}$ Department of Pediatrics, McMaster University, Hamilton, ON, Canada; \\ ${ }^{2}$ School of Rehabilitation Science, McMaster University, Hamilton, ON, Canada; \\ ${ }^{3}$ CanChild Centre for Childhood Disability Research, Department of Pediatrics, McMaster University, Hamilton, ON, Canada; \\ ${ }^{4}$ Brain Center Rudolf Magnus and Center of Excellence for Rehabilitation Medicine, \\ University Medical Center Utrecht and De Hoogstraat Rehabilitation, Utrecht, The Netherlands; \\ ${ }^{5}$ Department of Physical Medicine and Rehabilitation, University of Michigan-Medicine, Ann Arbor, MI, USA
}

\begin{abstract}
Objective To determine physical activity, sleep, and nutrition patterns in individuals with cerebral palsy (CP) and investigate the association of Gross Motor Function Classification System (GMFCS) and age with these health behaviors.

Methods A cross-sectional study was conducted in an outpatient setting. Participants included adolescents and adults with CP ( $\mathrm{n}=28$; GMFCS level I-V; mean age 35.1 \pm 14.4 years). An Exercise Questionnaire or Leisure Time Physical Activity Questionnaire was used to measure physical activity in adolescents and adults, respectively. Sleep quality was measured using the Pittsburgh Sleep Quality Index (PSQI). An adapted version of the PrimeScreen questionnaire was used to assess nutrition. Linear regression analyses were performed to investigate the association between GMFCS and age with physical activity, sleep, and nutrition.

Results The average total physical activity was $29.2 \pm 30.0 \mathrm{~min} /$ day. Seventy-five percent of the participants had poor sleep quality (PSQI score $>5$ ). Seventy-one percent reported "fair" eating behaviors; none reported "excellent" eating behaviors. Neither GMFCS nor age were significantly associated with PSQI score, PrimeScreen score, or total physical activity. A negative correlation existed between sleep quantity (hr/night) and PSQI score ( $\mathrm{r}=-0.66, \mathrm{p}=0.01)$.

Conclusion The triad of health components, consisting of physical activity, sleep, and nutrition, was not associated with GMFCS or age in our sample of 28 individuals with CP, suggesting that these three health behaviors should be assessed during clinical encounters of $\mathrm{CP}$ in adolescents and adults at all levels of the GMFCS.
\end{abstract}

Keywords Cerebral palsy, Exercise, Sleep, Nutrition assessment

Received September 17, 2019; Revised December 9, 2019; Accepted December 23, 2019; Published online July 28, 2020

Corresponding author: Patrick G. McPhee

Department of Pediatrics, McMaster University, 1280 Main St. West, Hamilton, ON L8S 4K1, Canada. Tel: +1-905-525-9140 (ext.21407), Fax: +1-905529-7687, E-mail: mcpheepg@mcmaster.ca

ORCID: Patrick G. McPhee (https://orcid.org/0000-0001-5613-9755); Olaf Verschuren (https://orcid.org/0000-0002-4443-5327); Mark D. Peterson (https:// orcid.org/0000-0002-5987-7128); Ada Tang (https://orcid.org/0000-0002-6641-4017); Jan Willem Gorter (https://orcid.org/0000-0002-3012-2119).

(c) This is an open-access article distributed under the terms of the Creative Commons Attribution Non-Commercial License (http://creativecommons.org/ licenses/by-nc/4.0) which permits unrestricted noncommercial use, distribution, and reproduction in any medium, provided the original work is properly cited. Copyright $\odot 2020$ by Korean Academy of Rehabilitation Medicine 


\section{INTRODUCTION}

Cerebral palsy (CP) is the most common pediatric physical disability, with an estimated prevalence ranging from 2.6 to 3.1 cases per 1,000 live births $[1,2]$. Although the majority of individuals with $\mathrm{CP}$ can expect similar life expectancies as the general population, except for those with greater severity and complexity [3], the vast majority of research in this population is focused on clinical symptom management in childhood and adolescence. Preventive medicine has become an emerging area since individuals with $\mathrm{CP}$ experience significant progressive functional declines, fatigue, diminished musculoskeletal mass and quality, excess adiposity, chronic physical inactivity, and increased risk for cardiovascular diseaseall of which increase with age [4-7]. Collectively, these factors place individuals with $\mathrm{CP}$ at a greater risk for secondary chronic conditions that further contribute to disability, diminished quality of life, loss of independence and diminished life expectancy [8].

There is an increasing interest in chronic disease surveillance [6] and promotion of behavior modification for people with CP, including physical activity [9], sleep [10], and nutrition [11]. However, there have been no systematic observational studies that have examined all three of these factors comprehensively in a cohort of individuals with CP. Physical activity has been studied most extensively, where individuals with $\mathrm{CP}$ have been reported to be less physically active than their typically developing peers [12,13], and ambulatory individuals with CP engage in more physical activity and decreased sedentary time than non-ambulatory individuals with CP [9]. Moreover, among ambulatory adults with $\mathrm{CP}$, younger age was associated with higher odds of being physically active [14].

Sleep issues are also a common occurrence among children with CP [10], which may be a product of intrinsic and extrinsic factors, such as muscle spasms or other forms of musculoskeletal pain and use of devices (e.g., orthosis, night worn splints, postural equipment), respectively [10]. Increasing awareness of the presence and the broad range of causes and consequences of inadequate sleep in people with $\mathrm{CP}$ throughout their lifespans is critical.

Few studies have examined the nutrition status or dietary habits of individuals with CP. One early study found that inactivity and malnutrition were relevant causes of abnormal body composition in 22 children with $\mathrm{CP}$ [15]. More recent research in 103 adolescents and young adults with CP without severe learning disabilities found that $27 \%$ indicated problems with nutrition [16]. To the best of our knowledge, no studies have examined nutrition in a broader age range of adults with $\mathrm{CP}$ in an outpatient setting.

Managing physical activity, sleep, and nutrition in a comprehensive fashion underscores the opportunity to encourage and promote the health and well-being of individuals with CP throughout their lifespans [11]. To guide clinicians, an important first step towards this goal is to observe and understand these behaviors in individuals with CP and determine if individuals with different functional levels experience different health behaviors. Therefore, the objectives of this study were to describe physical activity behaviors, sleep quantity and quality, and nutrition patterns in a cohort of individuals with $\mathrm{CP}$ and investigate the influence of disability severity and age on these components of health.

\section{MATERIALS AND METHODS}

This study is part of a larger on-going research investigation to examine the cardiovascular health and physical activity levels in adolescents and adults (min-max, 16-78 years) with $\mathrm{CP}$ [17]. A total of 53 individuals with $\mathrm{CP}$ who had participated in previous studies $[18,19]$ were contacted and invited to participate in this study. All individuals with CP were eligible for inclusion provided they were able to respond to questionnaires about physical activity, sleep, and nutrition with some degree of independence. The study design was explained to the participants and their caregivers prior to obtaining written consent. Participants were asked to complete the questionnaires on their own but assistance from a caregiver was allowed if needed. Approval from the Hamilton Integrated Research Ethics Board was obtained for the study (No. HiREB 2490).

\section{Demographic variables}

All interested participants were invited to the Vascular Dynamics Laboratory at McMaster University to undergo assessments of cardiovascular health as a component of the on-going research program. During this visit, supine body height, body mass recorded using a digital wheel- 
chair scale (Detecto Scales, FHD Series; Detecto, Webb City, MO, USA), and waist circumference were measured by the first author (PGM). Body mass index (BMI, $\mathrm{kg} / \mathrm{m}^{2}$ ) was calculated by dividing the participant's weight by their height squared. Waist circumference $(\mathrm{cm})$ was measured while the participant remained in a supine position, at the end of a normal expiration, and at $4 \mathrm{~cm}$ proximal to the umbilicus [5]. Gross Motor Function Classification System (GMFCS) level [20] was collected through self-report, and the type of motor impairment (spastic, dyskinetic, or mixed) and topographical distribution (unilateral or bilateral) were classified according to the Surveillance of Cerebral Palsy in Europe Guidelines [21].

\section{Physical activity}

Physical activity was quantified through selected questionnaires following the protocols within the studies for adolescents and adults with CP $[18,19]$. For adult participants, physical activity was measured using the Leisure Time Physical Activity Questionnaire for persons with Spinal Cord Injury (LTPAQ-SCI) [22]. Leisure Time Physical Activity (LTPA) is defined as intentional activities that people choose to do during their "free" time, such as exercising, playing sports, gardening, etc.; necessary physical activities, such as physiotherapy, grocery shopping, and/or wheeling for transportation were not considered LTPA [22]. Criterion validity and test-retest reliability have been reported for the LTPAQ-SCI in individuals with SCI [22]. Mild, moderate and heavy/vigorous intensity physical activity measured by the LTPAQ-SCI were associated with those captured using the Physical Activity Recall Assessment for people with SCI (PARA-SCI) [23]. While the PARA-SCI has been validated for use in adults with CP [9], the LTPAQ-SCI was chosen for the present study owing to its lower time requirement ( $<5$ minutes vs. 20-30 minutes for the PARA-SCI) [22]. Participants were asked to report the number of days and the number of minutes each day that they engaged in mild-, moderate-, and/or heavy/ vigorous-intensity LTPA during the previous 7 days. Participants were asked to provide information about both aerobic and strength training activities. The primary outcome of this questionnaire was the total number of minutes of LTPA at each intensity level performed during the previous 7 days. These totals were combined and divided by seven to give the average amount of physical activity performed per day (i.e., min/day). The average amount of moderate-to-vigorous physical activity (MVPA) per day was calculated after collecting data regarding the total moderate and heavy/vigorous intensity level activities for 7 days and dividing this value by seven.

For adolescent participants, physical activity was measured using the Exercise Questionnaire [24]. In this questionnaire, exercise was defined as activities that involved stretching, strengthening, or physical exertion [24]. The Exercise Questionnaire was developed in part from activities within the Previous Day Physical Activity Record, with the addition of activities that were appropriate for youth with CP following consultation with an expert group [24]. These additional items were confirmed by a pilot study of youth with $\mathrm{CP}$, which supported the content validity of the Exercise Questionnaire [24]. The questionnaire was designed to be completed by the adolescent with or without help from parents [24]. Test-retest reliability of the Exercise Questionnaire revealed no significant difference between two time points [24].

This questionnaire measures frequency, duration, and intensity of physical activity performed in the previous week. Total weekly duration (minute) of physical activity at all intensity levels (light, medium, or hard/vigorous) was calculated and divided by 7 to give an average amount (minutes) of physical activity performed per day.

\section{Sleep}

Sleep was measured using the Pittsburgh Sleep Quality Index (PSQI) [25]. The PSQI consists of 19 self-rated questions in seven component scores (sleep quality, sleep latency, sleep duration, habitual sleep efficiency, sleep disturbances, use of sleeping medications, and daytime dysfunction). Each component is scored from $0-3$, and the sum provides a global PSQI score (maximum score 21) where higher scores indicate poorer sleep quality. Scores $>5$ indicate poor sleep quality [25]. The PSQI takes 5-10 minutes to complete, and approximately 5 minutes to score. The PSQI has a high degree of internal consistency (Cronbach's $\alpha=0.83$ ) [25], and good testretest reliability (ICC $=0.85$ ) [25]. Regarding validity, the PSQI identifies between good and poor sleepers, and compares favorably with the criterion standard of sleep disorder diagnosis through polysomnography (t-tests showed no difference for sleep latency between the two measures) [25]. 


\section{Nutrition}

An adapted version of the PrimeScreen questionnaire was used to measure eating behaviors and food choices over the previous 6 months [26]. The questionnaire consists of 21 questions about the average frequency of consumption of specified foods and food groups during this time period. The questionnaire highlights intake of fruits, vegetables, whole and low-fat dairy products, whole grains, fish, poultry and red meat as well as other foods that are major contributors to fat intake [26]. The questionnaire includes examples of the most frequently consumed foods pertaining to a certain food group. For example, one specific question asks, "How often do you eat... dark green leafy vegetables (spinach, romaine lettuce, mesclun mix, kale, turnip greens, bok choy, swiss chard)". Frequency of consumption is categorized as: less than once per week, once per week, 2 to 4 times per week, nearly daily or daily, or twice or more per day with scores ranging from 0 to 4 for 11 questions, 0 to -4 for 9 questions, and -2 to 2 for 1 question. Scores from the 21 questions are totaled and eating behaviors are classified based on the following scores: $\geq 35$, excellent; 16-34, good; $1-15$, fair; and $\leq 0$, poor [26]. The PrimeScreen has been shown to have adequate reproducibility when compared with the semi-quantitative food frequency questionnaire (131 items), with an estimated correlation coefficient of 0.59 [26]. In a study assessing the reproducibility of the PrimeScreen, participants found it easy to understand and complete, with $87 \%$ of participants requiring $<10$ minutes to complete the questionnaire [26].

\section{Statistical analysis}

Statistical analyses were performed using Stata 13 statistical software package (Release 13; StataCorp LLC, College Station, TX, USA). Descriptive statistics were calculated and presented as means, standard deviations, minimum, lower quartiles, medians, upper quartiles, and maximum values for continuous variables, and as frequencies for dichotomous and categorical variables. All continuous variables were tested for normality using Shapiro-Wilk descriptive tests. Linear regression was performed to investigate the relationship between GMFCS level-dichotomized into community ambulatory (GMFCS I-II) and community non-ambulatory (GMFCS IIIV) (independent predictor) - and total physical activity (min/day), PSQI score, and PrimeScreen score, each as a dependent variable in separate models. Linear regression was also performed to investigate the association between age as a continuous variable (independent predictor) and total physical activity (min/day), PSQI score, and PrimeScreen score, again as dependent variables in separate models. Other correlations examined included BMI vs. PrimeScreen score, waist circumference vs. PrimeScreen score, and sleep quantity (hr/night) vs. PSQI score. A minimum criterion $\alpha$ level of $\mathrm{p} \leq 0.05$ was used to determine statistical significance.

\section{RESULTS}

\section{Participants}

Physical activity, PSQI, and PrimeScreen questionnaires were completed by 28 individuals with CP (mean age, $35.1 \pm 14.4$ years; min-max, $16-78$ years) from a database of 53 eligible individuals $[18,19]$. There was no significant difference in age, BMI and waist circumference between the 28 study participants and 25 non-study participants (i.e., $p>0.05$ ) (data not shown). Table 1 displays the participant characteristics. Due to the presence of an intrathecal baclofen pump or enteral feeding tube, it was not possible to obtain waist circumference measurements

Table 1. Participants' characteristics $(n=28)$

\begin{tabular}{|cc}
\hline \multicolumn{1}{c}{ Characteristic } & Value \\
\hline Sex & $13(46)$ \\
Male & $15(54)$ \\
Female & \\
Type & $23(82)$ \\
Spastic & $2(7)$ \\
Dyskinetic & $3(11)$ \\
Mixed & \\
\hline Distribution & $13(46)$ \\
\hline Unilateral & $15(54)$ \\
Bilateral & \\
\hline GMFCS & $7(25)$ \\
I & $6(21)$ \\
\hline II & $5(18)$ \\
\hline III & $7(25)$ \\
\hline IV & $3(11)$ \\
\hline V & \\
\hline
\end{tabular}

Values are presented as number (\%).

GMFCS, Gross Motor Function Classification System. 
Table 2. Results of variables

\begin{tabular}{lccccrr}
\hline & Mean \pm SD & Min & Low quartile & Median & Upper quartile & Max \\
\hline $\mathrm{Age}^{\mathrm{a})}(\mathrm{yr})$ & $35.1 \pm 14.4$ & 16.0 & 24.5 & 33.0 & 40.5 & 78.0 \\
$\mathrm{BMI}^{\mathrm{a}}\left(\mathrm{kg} / \mathrm{m}^{2}\right)$ & $26.1 \pm 8.1$ & 15.7 & 19.2 & 24.3 & 33.1 & 42.6 \\
Waist circumference $^{\mathrm{b})}(\mathrm{cm})$ & $84.0 \pm 19.7$ & 56.0 & 68.0 & 79.0 & 99.5 & 135.0 \\
Total PA (min/day) & $29.2 \pm 30.0$ & 0 & 8.6 & 19.3 & 42.5 & 125.0 \\
MVPA (min/day) & $13.5 \pm 18.8$ & 0 & 0 & 2.9 & 22.1 & 60.0 \\
PrimeScreen & $8.0 \pm 6.8$ & -4.0 & 3.0 & 8.0 & 12.0 & 21.0 \\
PSQI & $7.6 \pm 3.7$ & 1.0 & 5.5 & 8.0 & 9.5 & 16.0 \\
Sleep $^{\text {a) }}$ (hr/night) & $7.1 \pm 1.9$ & 4.0 & 6.0 & 6.5 & 8.0 & 12.0 \\
\hline
\end{tabular}

BMI, body mass index; PA, physical activity; MVPA, moderate-to-vigorous physical activity; PSQI, Pittsburgh Sleep Quality Index.

${ }^{a)}$ Data not normally distributed and therefore log transformed to assume normality. Raw values presented.

${ }^{\mathrm{b})} \mathrm{n}=27$.

at $4 \mathrm{~cm}$ proximal to the umbilicus of four participants. For three of these participants, waist circumference was instead measured at the border of the right anterior superior iliac crest; for one participant this was not possible as they were unable to move from the wheelchair to the testing bed.

Age, BMI, total physical activity, MVPA, and sleep (hr/ night) were not normally distributed (Shapiro-Wilk test $\mathrm{p}<0.05)$. The variables, including age, BMI, and sleep were log transformed to achieve statistical normality, with these transformed variables used in subsequent statistical analyses. Visual interpretation of the variables, including total physical activity and MVPA appeared to be normally distributed, and therefore, were included in the statistical analyses as original values (Table 2).

\section{Physical activity}

Total physical activity for participants was $29.2 \pm 30.0$ and 13.5 $\pm 18.8 \mathrm{~min} /$ day for MVPA. Overall, mean total physical activity (min/day) values by GMFCS level are displayed in Fig. 1. Twenty-two (78.6\%) participants engaged in $<150$ minutes of MVPA per week.

\section{Sleep}

Using the PSQI questionnaire, participants reported an average score of 7.6 \pm 3.7 . Twenty-one (75\%) participants had PSQI scores $>5$, indicating poor sleep quality, and 16 (57\%) obtained $<7$ hours of sleep per night. Mean PSQI scores and hours of sleep per night by GMFCS level are presented in Fig. 2A and 2B. Ten (36\%) participants indicated that they were using sleeping medication.

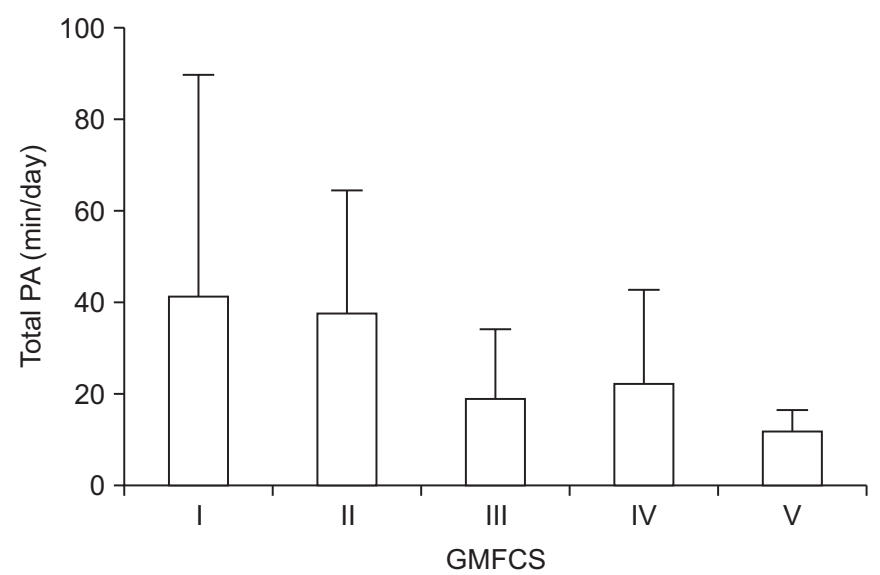

Fig. 1. Average total physical activity (PA) for each Gross Motor Function Classification System (GMFCS) level. Error bars indicate standard deviation.

\section{Nutrition}

Average scores for nutrition using an adapted version of the PrimeScreen questionnaire were $8.0 \pm 6.8$, which is in the fair range (score of 1-15). Only 4 (14\%) participants had "good" eating behaviors (score $>15$ ), whereas 4 (14\%) participants had "poor" eating behaviors (score of $\leq 0$ ). Mean PrimeScreen scores by GMFCS level are presented in Fig. 3.

\section{Predictors of sleep, nutrition, and physical activity}

GMFCS was not an independent predictor of PSQI score $(\beta=0.35 ; p=0.81 ; 95 \% C I,-2.55$ to 3.25$)$, PrimeScreen score ( $\beta=-0.93 ; p=0.72 ; 95 \% \mathrm{CI},-6.28$ to 4.42 ), or total physical activity ( $\mathrm{min} /$ day) ( $\beta=-20.5 ; \mathrm{p}=0.07 ; 95 \% \mathrm{CI},-42.9$ to 1.79 ). Similarly, age was not an independent predictor of 

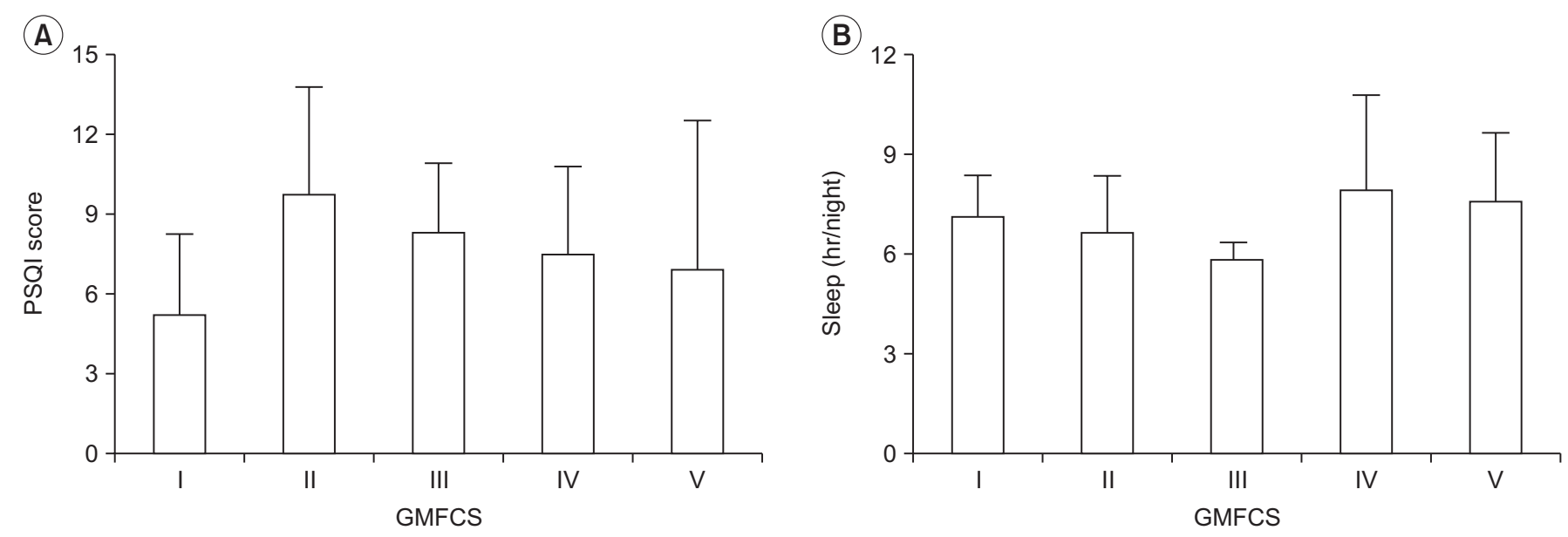

Fig. 2. (A) Average Pittsburgh Sleep Quality Index (PSQI) score for each Gross Motor Function Classification System (GMFCS) level. (B) Average sleep hours per night for each GMFCS level. Error bars indicate standard deviation.

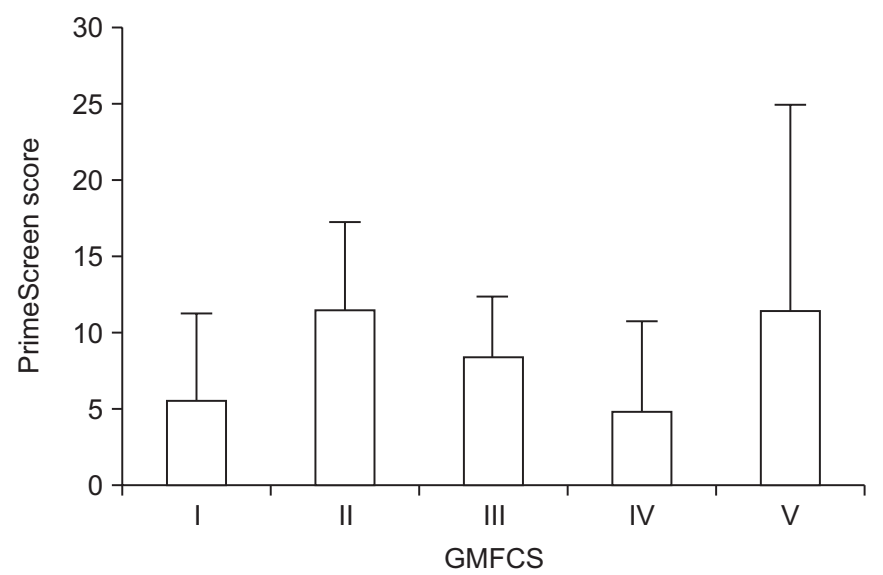

Fig. 3. Average PrimeScreen score for each Gross Motor Function Classification System (GMFCS) level. Error bars indicate standard deviation.

PSQI score $(\beta=1.74 ; p=0.35 ; 95 \% \mathrm{CI},-0.06$ to 0.14$)$, PrimeScreen score $(\beta=-1.63 ; \mathrm{p}=0.64 ; 95 \% \mathrm{CI},-0.24$ to 0.13$)$, or total physical activity ( $\mathrm{min} /$ day) $(\beta=-17.5 ; \mathrm{p}=0.25 ; 95 \%$ CI, -1.17 to 0.48$)$. We did not see any correlation between BMI and PrimeScreen score $(\mathrm{r}=0.15, \mathrm{p}=0.44)$ (Fig. 4A) or waist circumference and PrimeScreen score $(\mathrm{r}=0.03$, $\mathrm{p}=0.89$ ) (Fig. 4B). A negative correlation existed between sleep quantity (hours) and PSQI score ( $\mathrm{r}=-0.66, \mathrm{p}=0.01)$ (Fig. 4C).

\section{DISCUSSION}

Results from this study suggest that physical activity be- haviors, sleep quantity and quality, and nutrition of adolescents and adults with CP require attention from clinicians. Importantly, severity (GMFCS I-II vs. III-V) and age were not significantly associated with any component of health, suggesting that these three health variables should be assessed during clinical encounters of $\mathrm{CP}$ in all adolescents and adults, regardless of the GMFCS level.

Clinicians working with individuals with $\mathrm{CP}$ often presume that patients with higher functioning experience less health problems than those with lower functioning. Indeed, there is empirical evidence to support this presumption with regard to physical activity and sedentary behavior, as we have previously shown that adults with $\mathrm{CP}$ who have higher functioning engage in more habitual physical activity than those with lower functioning [9]. Regardless, overall physical activity is lower in adolescents [27] and adults [13] with CP compared with the general population. This is reflected in the present study, where almost $80 \%$ of participants reported engaging in $<150$ minutes of MVPA per week, which are the current recommendations by the World Health Organization for adults aged 18-64 years [28]. Individuals classified as GMFCS levels IV and V reported some involvement in light intensity activities, which is a great starting point that may lead to health gains [29]. However, individuals with GMFCS level V in this study did not engage in any moderate-to-vigorous physical activities.

Although some literature exists regarding a negative association between age and physical activity in adults with CP [14], age was not associated with physical activity 

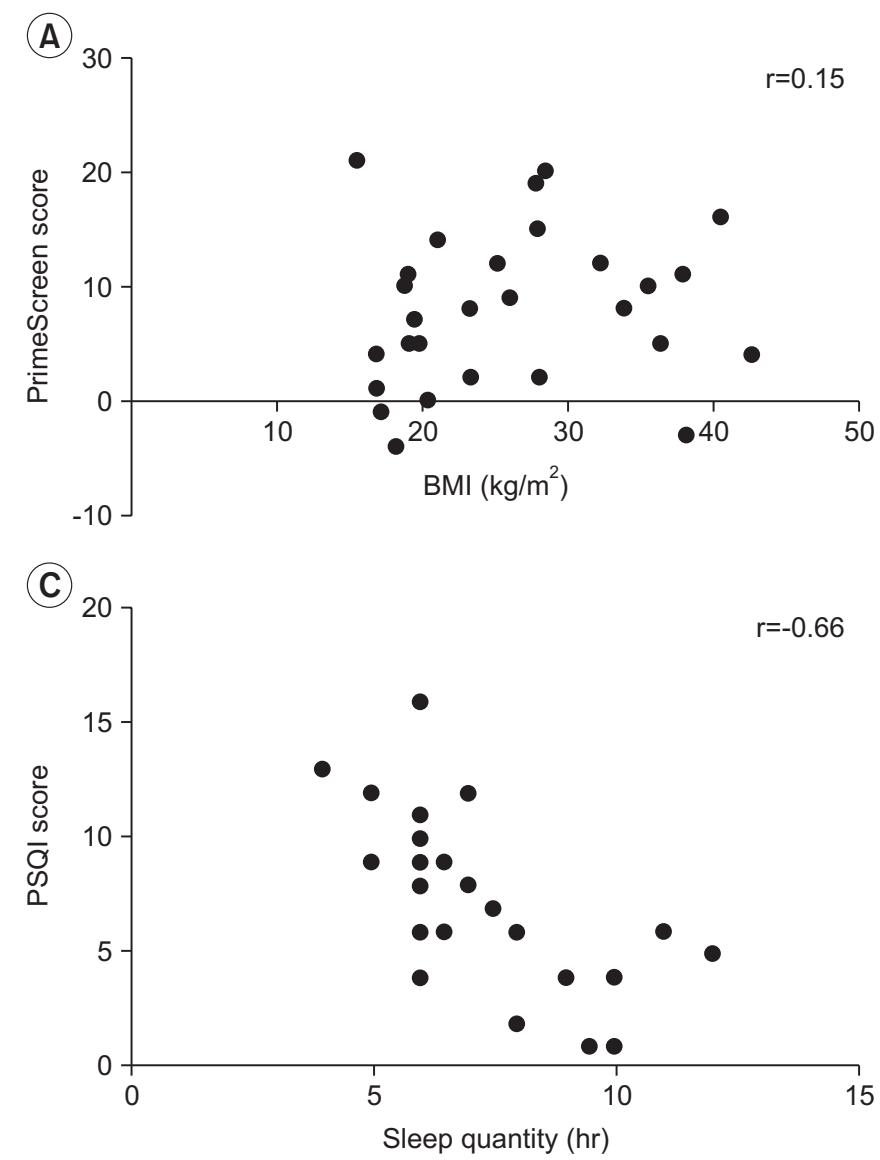

in this study. The reason for no association between age and physical activity could be due to a small sample size. Acknowledging not only the challenges and difficulties of those with excessive mobility limitations to engage in MVPA, but also proper nutrition and sleep management may also be critical for optimal health of individuals with CP [11]. Results from the present study illustrate, at the very least, assessment techniques for clinicians to capture and discuss with patients with $\mathrm{CP}$ the equally important health variables of physical activity, sleep, and nutrition.

It is well-known that sleep is an essential biological function with important roles in recovery, conservation of energy, and survival [30]. Insufficient sleep or patterns of sleep that interfere with physical and mental wellbeing can result from deprivation of sleep or decreased sleep quality. In the present study, $75 \%$ of the participants had poor sleep quality, indicated by a PSQI score $>5$. Moreover, more than half of the participants were getting less than the recommended 7 hours of sleep per night for adults [30]. When compared with the general

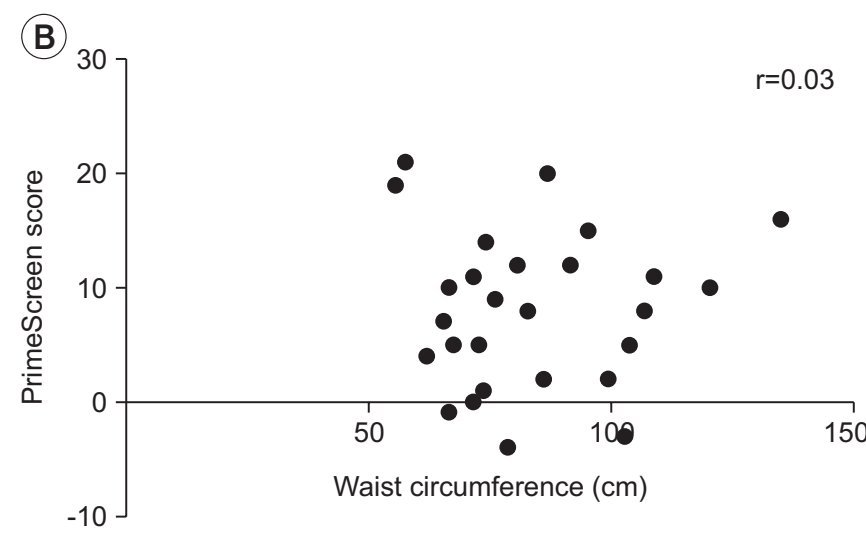

Fig. 4. (A) Pearson correlation between BMI and PrimeScreen score. (B) Pearson correlation between waist circumference and PrimeScreen score. (C) Pearson correlation between sleep quantity and PSQI score. BMI, body mass index; PSQI, Pittsburgh Sleep Quality Index.

population, 35.2\% of Americans obtain less than 7 hours of sleep per night [31]. We found a significant negative association between sleep quantity (hr/night) and PSQI score, which suggests that low sleep quantity is related to increased PSQI score in this group of individuals with CP. A simple solution may be to increase sleep quantity, but this approach minimizes the complexity of factors that potentially influence the hours of sleep per night for individuals with CP. For example, someone with spastic CP might awaken in the night frequently due to muscle spasms, which in turn, would affect sleep duration. There is some evidence in children and adults with $\mathrm{CP}$ that injections with botulinum toxin serotype A or oral baclofen, respectively, reduces spasticity and improves sleep [32,33]. However, it is important for clinicians to be mindful of the use of spasticity medications to assist with sleep, given that a component of the global PSQI score is use of sleeping medications. A person with CP may associate medical treatment of spasticity with sleep assistance, and in turn, score higher in that component of the PSQI. Thus, clinicians need to check if patients with 
$\mathrm{CP}$ are on sleeping pills and getting spasticity management when assessing sleep quality and quantity. Of the 10 participants in the present study who used medication to assist with sleeping, 8 had poor sleep quality.

There were no individuals with $\mathrm{CP}$ who reported excellent nutritional intake, again highlighting the important opportunities to improve in this area. We were able to use the feasible PrimeScreen questionnaire in adolescents and adults with CP in the present study to identify persons with suboptimal diets. The questionnaire has been shown to be easily understood and can be completed by most participants in $<10$ minutes [26], and can be readily applied in clinical settings. Future research may include the use of the PrimeScreen in combination with the Eating and Drinking Ability Classification System (EDACS) [34]. The EDACS is a system that describes functional eating and drinking ability in people with $\mathrm{CP}$ from 3 years of age and older [34]. Assessing nutrition and eating/ drinking ability together would afford an opportunity to understand the relationship between "what" and "how" individuals with $\mathrm{CP}$ eat.

Taken together, the triad of physical activity, sleep, and nutrition assessed in a comprehensive manner will allow clinicians to better understand modifiable behaviors that can prevent negative health consequences in adolescents and adults with CP. Although GMFCS was not a significant predictor of any one of the three health components, recommendations can be made for clinical care. Individuals who were GMFCS level I reported, on average, the highest amount of physical activity, lowest PSQI score, but the second lowest PrimeScreen score. These initial findings indicate, although in a small sample of individuals with $\mathrm{CP}$, that those who are GMFCS level I could benefit from dietary consultation to improve their overall health. On the other end of the spectrum, individuals with GMFCS level V had the highest PrimeScreen score, second lowest PSQI score, but the lowest total physical activity levels. This suggests that while physical activity should be promoted in this group, given the challenges to engage in physical activity for individuals with severe mobility limitations [29], clinicians may also strongly encourage continued good sleep and nutrition.

Future research investigating this health triad in persons with CP should do so in a larger sample across multiple sites, while assessing associations between measures of body composition and risk factors of cardio- vascular disease, which will allow for better generalizability and clinical implications of the findings.

\section{Limitations}

We present results for the triad of health in our study across all five levels of the GMFCS. Our findings should be interpreted with caution owing to the small number of participants. The use of subjective questionnaires to assess the components of health could have resulted in social desirable response bias [35]. Additionally, individuals volunteered to participate in this study, and therefore, could be more health conscious than a random sample from the community.

Another limitation in this study was the use of two questionnaires to assess physical activity. A notable difference between these questionnaires is that the Exercise Questionnaire asks participants whether or not they participated in specific exercises and their intensities in the last 7 days. However, there is an opportunity for participants to mention other exercises that they may have participated in that were not included in the questionnaire [24]. The LTPAQ-SCI asks participants about the frequency and duration of light, moderate, and heavy/ vigorous intensity activity performed in the previous 7 days [22]. Despite this difference, both afford an opportunity to quantify total and moderate to heavy/vigorous physical activity, allowing for a meaningful aggregation of responses from the two questionnaires. Finally, recent research suggests that BMI does not accurately estimate fat mass index in individuals with CP [36]. However, we did include measures of waist circumference, and a previous study did reveal a positive association between waist circumference and cardiometabolic disease risk in adults with $\mathrm{CP}$ [37].

\section{Conclusion}

The triad of health components, consisting of physical activity, sleep, and nutrition, were observed in a group of adolescents and adults with $\mathrm{CP}$ inclusive of all five levels of the GMFCS. Severity or age was not predictive of any component of health, emphasizing the importance of assessing the triad of health in adolescents and adults with $\mathrm{CP}$, regardless of the level of severity. 


\section{CONFLICT OF INTEREST}

Dr. Jan Willem Gorter holds the Scotiabank Chair in Child Health Research. The other authors declare that there is no conflict of interest.

\section{ACKNOWLEDGMENTS}

This project was inspired by a Letter to the Editor from 2016 [11]. We would like to acknowledge and thank the study participants for taking part in the study.

\section{AUTHOR CONTRIBUTION}

Conceptualization: McPhee PG, Verschuren O, Gorter JW. Methodology: McPhee PG, Verschuren O, Tang A, Gorter JW. Formal analysis: McPhee PG, Peterson MD. Writing - original draft: McPhee PG. Writing - review and editing: all authors. Approval of final manuscript: all authors.

\section{REFERENCES}

1. Christensen D, Van Naarden Braun K, Doernberg NS, Maenner MJ, Arneson CL, Durkin MS, et al. Prevalence of cerebral palsy, co-occurring autism spectrum disorders, and motor functioning: Autism and Developmental Disabilities Monitoring Network, USA, 2008. Dev Med Child Neurol 2014;56:59-65.

2. Maenner MJ, Blumberg SJ, Kogan MD, Christensen D, Yeargin-Allsopp M, Schieve LA. Prevalence of cerebral palsy and intellectual disability among children identified in two U.S. National Surveys, 2011-2013. Ann Epidemiol 2016;26:222-6.

3. Hutton JL. Outcome in cerebral palsy: life-expectancy. Paediatr Child Health (Oxford) 2008;18:419-22.

4. Shortland A. Muscle deficits in cerebral palsy and early loss of mobility: can we learn something from our elders? Dev Med Child Neurol 2009;51 Suppl 4:5963.

5. McPhee PG, Brunton LK, Timmons BW, Bentley T, Gorter JW. Fatigue and its relationship with physical activity, age, and body composition in adults with cerebral palsy. Dev Med Child Neurol 2017;59:367-73.

6. Peterson MD, Ryan JM, Hurvitz EA, Mahmoudi E. Chronic conditions in adults with cerebral palsy.
JAMA 2015;314:2303-5.

7. Peterson MD, Kamdar N, Hurvitz EA. Age-related trends in cardiometabolic disease among adults with cerebral palsy. Dev Med Child Neurol 2019;61:484-9.

8. Kruse M, Michelsen SI, Flachs EM, Bronnum-Hansen $\mathrm{H}$, Madsen M, Uldall P. Lifetime costs of cerebral palsy. Dev Med Child Neurol 2009;51:622-8.

9. Claridge EA, McPhee PG, Timmons BW, Martin Ginis KA, Macdonald MJ, Gorter JW. Quantification of physical activity and sedentary time in adults with cerebral palsy. Med Sci Sports Exerc 2015;47:1719-26.

10. Verschuren O, Gorter JW, Pritchard-Wiart L. Sleep: an underemphasized aspect of health and development in neurorehabilitation. Early Hum Dev 2017;113:1208.

11. Verschuren O, McPhee P, Rosenbaum P, Gorter JW. The formula for health and well-being in individuals with cerebral palsy: physical activity, sleep, and nutrition. Dev Med Child Neurol 2016;58:989-90.

12. Maher CA, Williams MT, Olds T, Lane AE. Physical and sedentary activity in adolescents with cerebral palsy. Dev Med Child Neurol 2007;49:450-7.

13. Ryan JM, Crowley VE, Hensey O, Broderick JM, McGahey A, Gormley J. Habitual physical activity and cardiometabolic risk factors in adults with cerebral palsy. Res Dev Disabil 2014;35:1995-2002.

14. Maltais DB, Dumas F, Boucher N, Richards CL. Factors related to physical activity in adults with cerebral palsy may differ for walkers and nonwalkers. Am J Phys Med Rehabil 2010;89:584-97.

15. Berg K, Isaksson B. Body composition and nutrition of school children with cerebral palsy. Acta Paediatr Scand Suppl 1970;Suppl 204:41-52.

16. Donkervoort M, Roebroeck M, Wiegerink D, van der Heijden-Maessen H, Stam H; Transition Research Group South West Netherlands. Determinants of functioning of adolescents and young adults with cerebral palsy. Disabil Rehabil 2007;29:453-63.

17. McPhee PG, MacDonald MJ, Cheng JL, Dunford EC, Gorter JW. Emerging evidence for accelerated ageing and cardiovascular disease in individuals with cerebral palsy. J Rehabil Med 2019;51:525-31.

18. Martin AA, Cotie LM, Timmons BW, Gorter JW, Macdonald MJ. Arterial structure and function in ambulatory adolescents with cerebral palsy are not different from healthy controls. Int J Pediatr 2012;2012:168209. 
19. McPhee PG, Gorter JW, Cotie LM, Timmons BW, Bentley T, MacDonald MJ. Associations of non-invasive measures of arterial structure and function, and traditional indicators of cardiovascular risk in adults with cerebral palsy. Atherosclerosis 2015;243:462-5.

20. Palisano RJ, Rosenbaum P, Bartlett D, Livingston $\mathrm{MH}$. Content validity of the expanded and revised Gross Motor Function Classification System. Dev Med Child Neurol 2008;50:744-50.

21. Surveillance of Cerebral Palsy in Europe. Surveillance of cerebral palsy in Europe: a collaboration of cerebral palsy surveys and registers. Surveillance of Cerebral Palsy in Europe (SCPE). Dev Med Child Neurol 2000;42:816-24.

22. Martin Ginis KA, Phang SH, Latimer AE, ArbourNicitopoulos KP. Reliability and validity tests of the leisure time physical activity questionnaire for people with spinal cord injury. Arch Phys Med Rehabil 2012;93:677-82.

23. Latimer AE, Ginis KA, Craven BC, Hicks AL. The physical activity recall assessment for people with spinal cord injury: validity. Med Sci Sports Exerc 2006;38:208-16.

24. Brunton LK, Bartlett DJ. Description of exercise participation of adolescents with cerebral palsy across a 4-year period. Pediatr Phys Ther 2010;22:180-7.

25. Buysse DJ, Reynolds CF 3rd, Monk TH, Berman SR, Kupfer DJ. The Pittsburgh Sleep Quality Index: a new instrument for psychiatric practice and research. Psychiatry Res 1989;28:193-213.

26. Rifas-Shiman SL, Willett WC, Lobb R, Kotch J, Dart C, Gillman MW. PrimeScreen, a brief dietary screening tool: reproducibility and comparability with both a longer food frequency questionnaire and biomarkers. Public Health Nutr 2001;4:249-54.

27. Nooijen CF, Slaman J, Stam HJ, Roebroeck ME, BergEmons RJ; Learn2Move Research Group. Inactive and sedentary lifestyles amongst ambulatory adolescents and young adults with cerebral palsy. J Neuroeng Rehabil 2014;11:49.

28. World Health Organization. Physical activity [Internet]. Geneva, Switzerland: World Health Organiza- tion; 2017 [cited 2020 Jun 15]. Available from: https:// www.who.int/news-room/fact-sheets/detail/physical-activity.

29. Verschuren O, Peterson MD, Balemans AC, Hurvitz EA. Exercise and physical activity recommendations for people with cerebral palsy. Dev Med Child Neurol 2016;58:798-808.

30. Mukherjee S, Patel SR, Kales SN, Ayas NT, Strohl KP, Gozal D, et al. An official American Thoracic Society statement: the importance of healthy sleep. Recommendations and future priorities. Am J Respir Crit Care Med 2015;191:1450-8.

31. Centers for Disease Control and Prevention. Short sleep duration among US adults [Internet]. Atlanta, GA: Centers for Disease Control and Prevention; 2017 [cited 2020 Jun 15]. Available from: https://www.cdc. gov/sleep/data_statistics.html.

32. Binay Safer V, Ozbudak Demir S, Ozkan E, Demircioglu Guneri F. Effects of botulinum toxin serotype A on sleep problems in children with cerebral palsy and on mothers sleep quality and depression. Neurosciences (Riyadh) 2016;21:331-7.

33. McCarty SF, Gaebler-Spira D, Harvey RL. Improvement of sleep apnea in a patient with cerebral palsy. Am J Phys Med Rehabil 2001;80:540-2.

34. Sellers D, Mandy A, Pennington L, Hankins M, Morris C. Development and reliability of a system to classify the eating and drinking ability of people with cerebral palsy. Dev Med Child Neurol 2014;56:245-51.

35. van de Mortel TF. Faking it: social desirability response bias in self-report research. Aust J Adv Nurs 2008;25(4):40-8.

36. Whitney DG, Miller F, Pohlig RT, Modlesky CM. BMI does not capture the high fat mass index and low fatfree mass index in children with cerebral palsy and proposed statistical models that improve this accuracy. Int J Obes 2019;43:82-90.

37. Ryan JM, Crowley VE, Hensey O, McGahey A, Gormley J. Waist circumference provides an indication of numerous cardiometabolic risk factors in adults with cerebral palsy. Arch Phys Med Rehabil 2014;95:15406. 